\title{
A percepção de apoio social e a sintomatologia depressiva em mulheres jovens atendidas em uma Unidade de Saúde da Família
}

\author{
The perception of social support and depressive symptomatology in young women assisted at a \\ Family Health Center
}

\section{La percepción de apoyo social y la sintomatología depresiva en mujeres jóvenes atendidas en una Unidad de Salud de la Familia}

Viviane Vedovato Silva Rocha. Programa de Residência Multiprofissional em Atenção Integral à Saúde, Faculdade de Medicina de Ribeirão Preto, Universidade de São Paulo (USP). Ribeirão Preto, SP, Brasil. vivi_vedovato @ hotmail.com (Autora correspondente)

Cassiana Morais de Oliveira. Hospital das Clínicas da Faculdade de Medicina de Ribeirão Preto, Departamento de Neurociências e Ciências do Comportamento, Serviço de Psicologia. Ribeirão Preto, SP, Brasil. cassianamoraes@gmail.com Rosana Shuhama. Faculdade de Medicina de Ribeirão Preto, Universidade de São Paulo (USP). Ribeirão Preto, SP, Brasil. roshuhama@gmail.com

\section{Resumo}

Objetivo: O estudo buscou identificar a percepção de apoio social em 55 mulheres de 20 a 39 anos, cadastradas em uma Unidade de Saúde da Família de uma cidade de médio porte localizada no interior do Estado de São Paulo, verificando possível associação com sintomas depressivos e características sociodemográficas, clínicas e de configuração familiar. Métodos: Estudo observacional de corte transversal realizado com auxílio de um Roteiro de Entrevista Estruturado, Inventário de Depressão de Becke Escala de Apoio Social da Medical Outcomes Study. Os dados foram analisados por meio de estatística descritiva e submetidos a testes não paramétricos. Resultados: Os resultados indicaram sintomas sugestivos de depressão em $25,5 \%$ da amostra; correlação negativa entre as dimensões de apoio social e depressão, bem como evidências de associação da percepção de apoio social em relação às características sociodemográficas (renda) e de configuração familiar (estado civil e número de filhos). Não foram encontradas evidências estatísticas na associação com as características clínicas. Conclusão: Estes resultados corroboram os achados da literatura nacional e internacional, indicando a necessidade do cuidado com mulheres jovens independentemente da vulnerabilidade específica.

\section{Abstract}

Objective: The study aimed to identify the perception of social support in 55 women aged between 20 and 39 , enrolled in a Family Health Center of a medium-size town in São Paulo State/Brazil, verifying its association with depressive symptoms and sociodemographic, clinics and family configuration characteristics. Methods: This cross-sectional observational study was carried out with the aid of a Structured Interview, Beck Depression Inventory and Social Support Scale of Medical Outcomes Study. Data were analyzed through descriptive statistics and non-parametric tests. Results: The results indicated suggestive symptoms of depression in $25.5 \%$ of the sample; negative correlation between the dimensions of social support and depression, as well as association of perceived social support in relation to the sociodemographic (income) and family configuration (marital status and number of children) characteristics. There was no statistical evidence of association with clinical characteristics. Conclusion: These results corroborate the findings of national and international literature, indicating the need for care with women regardless of specific vulnerability.
Palavras-chave:

Apoio Social

Depressão

Mulheres

Atenção Primária à Saúde

\section{Keywords:}

Social Support

Depression

Women

Primary Health Care

Fonte de financiamento:

declaram não haver.

Parecer CEP:

1.141.361 (CSE-FMRP-USP), aprovado 07/07/2015

Conflito de interesses: declaram não haver.

Procedência e revisão por pares: revisado por pares. Recebido em: 07/03/2016. Aprovado em: 20/07/2016. 


\section{Resumen}

Objetivo: El estudio intentó identificar la percepción de apoyo social en 55 mujeres de 20 a 39 años, registrados en una Unidad de Salud de la Familia de una ciudad mediana en el estado de São Paulo/Brasil, comprobando la posible asociación con síntomas depresivos y características sociodemográficas, clínicas y configuración familiar. Métodos: Estudio observacional transversal realizado con la ayuda de una Entrevista Estructurada, Inventario de Depresión de Beck y la Escala de Apoyo Social del Medical Outcomes Study. Los datos fueron analizados mediante estadísticas descriptivas y pruebas no paramétricas. Resultados: Los resultados indicaron síntomas sugestivos de depresión en el $25,5 \%$ de la muestra; correlación negativa entre las dimensiones de apoyo social y depresión, así como evidencia de la asociación del apoyo social percibido en relación con las características sociodemográficas (ingresos) y configuración familiar (estado civil y número de hijos). No se encontró evidencia estadística en asociación con las características clínicas. Conclusión: Estos resultados corroboran los hallazgos de la literatura nacional e internacional, lo que indica la necesidad de ver a las mujeres jóvenes sin importar la vulnerabilidad específica.
Palabras clave:

Apoyo Social

Depresión

Mujeres

Atención Primaria de Salud

\section{Introdução}

O ser humano requer, em diferentes momentos da vida, a inserção em contextos cujas relações sejam fontes de apoio ou suporte social. Considerado um construto multifacetado e complexo, o apoio social pode ser compreendido quanto ao grau de satisfação do indivíduo com a disponibilidade e a qualidade das funções provindas das relações interpessoais. ${ }^{1-3}$ Refere-se à percepção que um indivíduo tem ao fazer parte de uma rede complexa na qual se pode dar e receber afeto ou ajuda. ${ }^{4}$ Relaciona-se aos recursos postos à disposição por outras pessoas em situações de necessidade. ${ }^{5}$

Estudos realizados na década de 1970 já sugeriam que a ruptura de laços sociais afeta diretamente os sistemas de defesa do organismo, tornando o indivíduo mais suscetível a doenças. ${ }^{6}$ Além dos agravos físicos, o apoio social também tem sido relacionado com o campo da saúde mental, ao considerar que o risco de acometimento de doenças como depressão tende a ser menor na presença de apoio social, ao passo que a ausência deste apoio aumenta a probabilidade de sua ocorrência. ${ }^{7}$

Apontado como um problema de saúde mental cada vez mais presente na sociedade, o transtorno depressivo maior apresenta prevalência de 12 meses em torno de 7\% nos Estados Unidos, enquanto no Brasil, apesar da fragilidade na produção de dados epidemiológicos atuais e abrangentes, os índices variam entre $8 \%$ e 12\%.8,9 Também há evidências de que a doença afete até três vezes mais mulheres que homens e, apesar de seu início ocorrer em qualquer idade, a incidência parece atingir seu pico na segunda década de vida, sendo três vezes maior em indivíduos de 18 a 29 anos em relação àqueles com mais de 60 anos. ${ }^{8}$

Considerado fundamental para amenizar estressores cotidianos, principalmente em situações que envolvem mudanças psicossociais e fisiológicas, o apoio social sólido desde a juventude favorece o desenvolvimento de atitude positiva e emissão de condutas mais adaptativas, podendo contribuir para a manutenção da saúde e favorecer o prognóstico de morbidades, inclusive a depressão.4,6,10,11

Tanto o apoio social quanto os sintomas depressivos podem ser melhor avaliados na Atenção Primária à Saúde por ser a porta de entrada do Sistema Único de Saúde, bem como proporcionar maior proximidade entre os profissionais e o contexto social dos usuários. Tendo em vista a escassez de estudos sobre o tema com mulheres jovens adultas, o presente estudo buscou identificar a percepção do apoio social e possíveis associações com sintomas depressivos, características sociodemográficas, clinicas e de configuração familiar dessas mulheres atendidas em uma Unidade de Saúde da Família. 


\section{Métodos}

Trata-se de um estudo observacional de corte transversal com uma amostra de conveniência composta por 55 mulheres de 20 a 39 anos regularmente cadastradas em uma Unidade de Saúde da Família (USF) de uma cidade de médio porte localizada no interior do Estado de São Paulo. Foram excluídas participantes com algum tipo de deficiência mental/cognitiva/auditiva/visual relatada ou observada pela pesquisadora que impossibilitasse a realização da entrevista.

A coleta de dados foi realizada individualmente nas dependências da USF ou no domicílio da participante, mediante assinatura de Termo de Consentimento Livre e Esclarecido e condições mínimas de privacidade. Os instrumentos utilizados consistiram em um Roteiro de Entrevista Estruturado elaborado pelas pesquisadoras, o Inventário de Depressão de Beck - BDI adaptado e validado para o Brasil ${ }^{12,13}$ e a Escala de Apoio Social utilizada na Medical Outcomes Study adaptada e validada para o Brasil. ${ }^{3,6}$

O Roteiro de Entrevista Estruturado consistiu em itens relacionados às características sociodemográficas, clínicas e de configuração familiar. O BDI, por sua vez, é um instrumento de autoaplicação não diagnóstico, composto por 21 itens, cujo objetivo é medir a intensidade da depressão em indivíduos a partir de 10 anos. Para uma melhor análise estatística deste estudo, foi necessário agrupar e reclassificar as duas últimas categorias deste instrumento (Mínimo, Leve, Moderado e Grave) em uma nova categoria nomeada "Sugestivo de depressão", em razão da baixa frequência de participantes na categoria Grave $(n=1)$.

A Escala de Apoio Social é um instrumento composto por 19 itens em uma escala Likert de cinco pontos $(1=$ nunca e 5 = sempre), que abrange três dimensões de apoio social: Material (provisão de recursos práticos e ajuda material); Afetiva/Interação Social Positiva (demonstrações físicas de amor e afeto, bem como ter pessoas com quem relaxar e divertir-se) e Emocional/Informação (habilidade da rede social em satisfazer as necessidades individuais em relação a problemas emocionais, bem como contar com pessoas que aconselhem, informem e orientem). A média das três dimensões podem ser sintetizadas em uma dimensão Global.

Para o tratamento estatístico dos dados, foi utilizado o programa Statistical Package for the Social Sciences (SPSS), versão 17.0 para Windows. Os escores das dimensões de apoio social foram descritos em suas médias e desvios-padrão, sendo submetidos aos testes não paramétricos Kruskal-Wallis e Mann-Whitney. As correlações foram analisadas por meio do coeficiente de Spearman, considerando os seguintes parâmetros de magnitude: ${ }^{14}$ fraca (menor ou igual a 0,3 ), moderada (maior que 0,3 e menor que 0,6) e forte (a partir de 0,6). Para todas as análises, foi considerado nível de significância de 5\%.

O projeto foi submetido ao Comitê de Ética em Pesquisa do Centro de Saúde Escola da Faculdade de Medicina de Ribeirão Preto - Universidade de São Paulo, respeitando a Resolução 466 de 2012 do Conselho Nacional de Saúde para pesquisa com seres humanos, sendo aprovado em 07/07/2015 (№ Parecer: 1.141.361). Os casos identificados com sintomas sugestivos de depressão foram discutidos com a equipe de saúde responsável e encaminhados para serviço de assistência melhor indicado. 


\section{Resultados}

A caracterização sociodemográfica demonstra que a maioria das participantes tinha Ensino Médio $(50,9 \%)$, profissão remunerada $(65,5 \%)$, renda familiar entre um a seis salários mínimos $(85,4 \%)$ e crença religiosa $(85,4 \%$, sendo $54,5 \%$ praticante). Quanto à configuração familiar, grande parte residia com até cinco pessoas $(89,1 \%)$, tinha companheiro $(60,0 \%$, sendo $52,7 \%$ amasiada/casada), relacionamento amoroso entre dois e dez anos $(41,8 \%)$ e até dois filhos $(87,3 \%)$.

Em relação às características clínicas, a maioria não apresentava hábitos etilistas $(60 \%)$ e tabagistas $(89,1 \%)$, não realizava ou realizava em baixa frequência exercício físico durante a semana $(58,2 \%)$, não participava de grupos de promoção à saúde oferecidos pela USF $(98,2 \%)$ e não apresentava agravos prévios $(61,8 \%)$. Entre aquelas que apresentavam algum agravo prévio, notou-se que $27,2 \%$ estavam relacionados a problemas de saúde mental.

No que se refere à ocorrência de sintomas depressivos, observou-se a seguinte distribuição das participantes entre as categorias do BDI: Mínimo ( $n=23$ - 41,8\%); Leve ( $n=18-32,7 \%)$ e Sugestivo de Depressão (n=14 - 25,5\%), apresentando escore médio de 14,2 ( $\pm 10,0)$. Quanto à percepção do apoio social, a Tabela 1 sintetiza os escores obtidos em cada dimensão da escala.

Tabela 1. Percepção do Apoio Social $(n=55)$.

\begin{tabular}{lccccccc}
\hline Dimensões de Apoio Social & M & DP & Mínimo & 1 $^{\circ}$ Quartil & Mediana & $3^{\circ}$ Quartil & Máximo \\
\hline Material & 82,6 & $\pm 20,4$ & 20,0 & 75,0 & 90,0 & 100,0 & 100,0 \\
Afetivo/Interação Social Positiva & 83,8 & $\pm 17,7$ & 45,7 & 65,7 & 88,6 & 100,0 & 100,0 \\
Emocional/Informação & 75,8 & $\pm 23,2$ & 30,0 & 55,0 & 82,5 & 97,5 & 100,0 \\
Global & 80,4 & $\pm 18,2$ & 38,9 & 66,3 & 84,2 & 97,9 & 100,0 \\
\hline
\end{tabular}

DP: Desvio-padrão; M: Média.

Observou-se uma correlação negativa entre a percepção de apoio social e os sintomas depressivos, isto é, quanto maior a percepção de apoio social, menor a gravidade de sintomas depressivos (Material: Rho= -0,287 e p=0,034; Afetivo/Interação Social Positiva: Rho= -0,492 e p<0,001; Emocional/Informação: Rho $=-0,528$ e $p<0,001$; Global: Rho= $-0,545$ e $p<0,001)$.

De acordo com o Gráfico 1, houve diferença significativa na percepção de apoio social de mulheres sem índicios de depressão (categoria Mínimo) em relação àquelas com sintomas leves e sugestivos de Depressão, exceto na dimensão Material $(p=0,053)$. Notou-se também que, apesar da percepção de apoio social ter sido mais elevada em mulheres com sintomas leves em relação àquelas com sintomas sugestivos de depressão, essa diferença não foi estatísticamente significativa em todas as dimensões $(p>0,05)$.

Quanto às comparações entre as dimensões de apoio social e as características sociodemográficas, houve diferença significativa apenas para a variável renda, conforme exposto na Tabela 2. Mulheres com renda entre 1 a 3 salários mínimos, quando comparado com as de 4 a 6 salários mínimos, apresentaram 


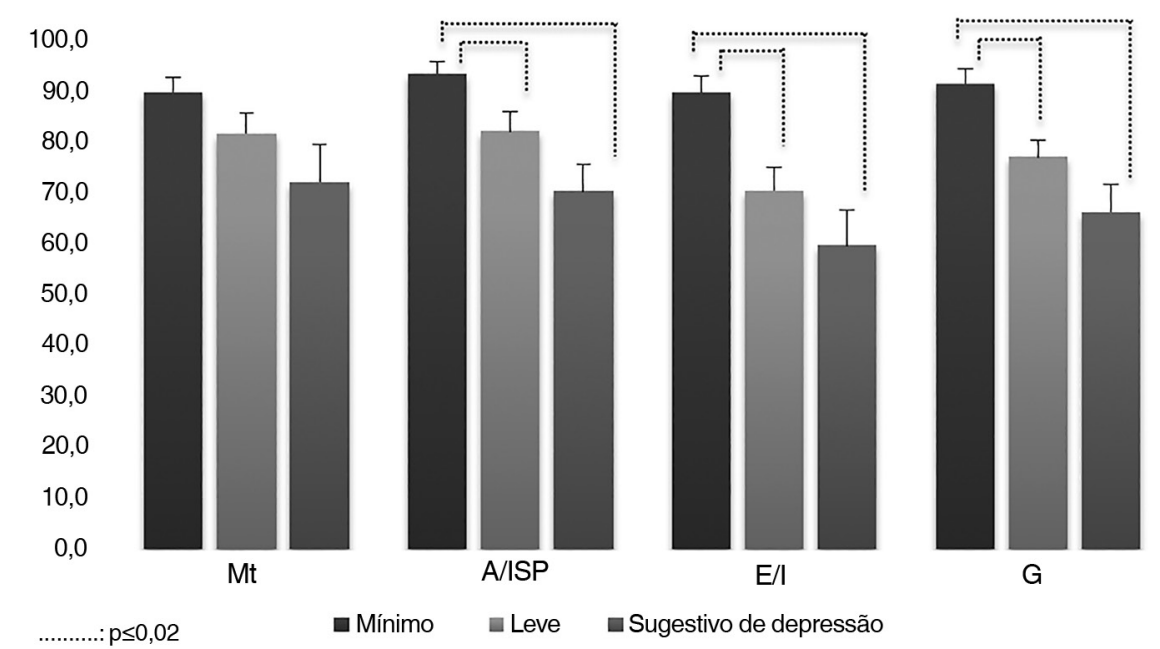

Gráfico 1. Comparação entre percepção de apoio social e nível de sintomas depressivos ( $n=55)$. Mt: Material; A/ISP: Afetivo/Interação Social Positiva; E/l: Emocional/Informação; G: Global.

menor percepção de apoio nas dimensões Material $(p=0,014)$, Emocional/Informação $(p=0,030)$ e Global $(p=0,040)$. Não foram encontrados resultados significativos nas demais comparações, sendo desconsideradas aquelas envolvendo os grupos de até 1 salário mínimo e 7 ou mais salários mínimos, devido à baixa representatividade de participantes.

Tabela 2. A percepção de apoio social segundo as características sociodemográficas.

\begin{tabular}{|c|c|c|c|c|c|c|c|c|c|c|}
\hline \multirow{2}{*}{ Variáveis Sociodemográficas } & \multirow{2}{*}{$\mathbf{n}$} & \multirow{2}{*}{$\%$} & \multicolumn{2}{|c|}{ Mt } & \multicolumn{2}{|c|}{ A/ISP } & \multicolumn{2}{|c|}{ E/I } & \multicolumn{2}{|c|}{ G } \\
\hline & & & $\mathbf{M}$ & (DP) & $\mathbf{M}$ & (DP) & $\mathbf{M}$ & (DP) & $\mathbf{M}$ & (DP) \\
\hline \multicolumn{11}{|l|}{ Faixa etária } \\
\hline 20 a 29 anos & 22 & 40,0 & 87,0 & $( \pm 16,8)$ & 82,6 & $( \pm 17,3)$ & 73,2 & $( \pm 21,6)$ & 80,0 & $( \pm 17,2)$ \\
\hline 30 a 39 anos & 33 & 60,0 & 79,7 & $( \pm 22,2)$ & 84,7 & $( \pm 18,1)$ & 77,6 & $( \pm 24,4)$ & 80,6 & $( \pm 19,1)$ \\
\hline \multicolumn{11}{|l|}{ Escolaridade } \\
\hline Ensino Fundamental & 7 & 12,7 & 79,3 & $( \pm 21,1)$ & 80,0 & $( \pm 20,5)$ & 77,5 & $( \pm 22,0)$ & 78,8 & $( \pm 18,5)$ \\
\hline Ensino Médio & 28 & 50,9 & 82,9 & $( \pm 19,6)$ & 88,4 & $( \pm 15,0)$ & 77,1 & $( \pm 21,4)$ & 82,8 & $( \pm 17,0)$ \\
\hline Ensino Superior & 20 & 36,4 & 82,5 & $( \pm 22,1)$ & 78,9 & $( \pm 19,3)$ & 73,5 & $( \pm 26,8)$ & 77,6 & $( \pm 20,1)$ \\
\hline \multicolumn{11}{|l|}{ Profissão Remunerada } \\
\hline Não & 19 & 34,5 & 80,5 & $( \pm 24,5)$ & 82,3 & $( \pm 18,8)$ & 70,3 & $( \pm 24,1)$ & 77,4 & $( \pm 19,9)$ \\
\hline Sim & 36 & 65,5 & 83,8 & $( \pm 18,1)$ & 84,7 & $( \pm 2,9)$ & 78,8 & $( \pm 17,3)$ & 82,0 & $( \pm 17,4)$ \\
\hline \multicolumn{11}{|l|}{ Renda Familiar (s. m.) } \\
\hline Até 1 & 2 & 3,6 & 100,0 & - & 100,0 & - & 100,0 & - & 100,0 & - \\
\hline 1 a 3 & 24 & 43,6 & 78,1 & $( \pm 19,3)$ & 81,7 & $( \pm 18,6)$ & 69,0 & $( \pm 22,7)$ & 76,0 & $( \pm 17,7)$ \\
\hline 4 a 6 & 23 & 41,8 & 89,3 & $( \pm 16,5)$ & 85,8 & $( \pm 17,5)$ & 82,7 & $( \pm 21,7)$ & 85,3 & $( \pm 17,0)$ \\
\hline 7 ou mais & 6 & 10,9 & 69,2 & $( \pm 30,6)$ & 79,5 & $( \pm 16,6)$ & 68,8 & $( \pm 24,9)$ & 72,8 & $( \pm 21,3)$ \\
\hline \multicolumn{11}{|l|}{ Religião } \\
\hline Não possui & 8 & 14,5 & 85,6 & $( \pm 13,7)$ & 83,6 & $( \pm 18,4)$ & 77,2 & $( \pm 24,4)$ & 81,3 & $( \pm 19,0)$ \\
\hline Possui e frequenta & 30 & 54,5 & 78,3 & $( \pm 23,6)$ & 84,1 & $( \pm 17,3)$ & 74,5 & $( \pm 24,1)$ & 78,8 & $( \pm 18,8)$ \\
\hline Possui e não frequenta & 17 & 30,9 & 88,8 & $( \pm 15,3)$ & 83,5 & $( \pm 18,9)$ & 77,5 & $( \pm 22,3)$ & 82,7 & $( \pm 17,6)$ \\
\hline Total & 55 & 100,0 & - & & - & & - & & - & \\
\hline
\end{tabular}

A/ISP: Afetivo/Interação Social Positiva; DP: Desvio-padrão; E/l: Emocional/Informação; G: Global; M: Média; Mt: Material; S.M.: Salário Mínimo. 
No que se refere às comparações entre as dimensões de apoio social e às características de configuração familiar, observou-se que as solteiras apresentaram maior percepção de apoio social Material em relação às casadas $(p=0,047)$ e divorciadas $(p=0,035$, no entanto, esta categoria de estado civil deve ser considerada com ressalvas, pois possui baixa representatividade). Além disso, mulheres com maior número de filhos apresentaram menor percepção de apoio Material $(p=0,024)$, sendo a força desta correlação fraca (rho= -0,305). Não foram encontrados resultados significativos nas demais comparações, conforme apresentado na Tabela 3.

Tabela 3. A percepção do apoio social segundo as características de configuração familiar.

\begin{tabular}{|c|c|c|c|c|c|c|c|c|c|c|}
\hline \multirow{2}{*}{ Configuração familiar } & \multirow{2}{*}{$\mathbf{n}$} & \multirow{2}{*}{$\%$} & \multicolumn{2}{|c|}{ Mt } & \multicolumn{2}{|c|}{ A/ISP } & \multicolumn{2}{|c|}{$E / I$} & \multicolumn{2}{|c|}{ G } \\
\hline & & & $\mathbf{M}$ & (DP) & $\mathbf{M}$ & (DP) & $\mathbf{M}$ & (DP) & $\mathbf{M}$ & (DP) \\
\hline \multicolumn{11}{|l|}{ № de moradores } \\
\hline 1 a 2 & 24 & 43,6 & 84,8 & $( \pm 19,1)$ & 82,6 & $( \pm 16,7)$ & 74,6 & $( \pm 22,6)$ & 79,7 & $( \pm 16,9)$ \\
\hline 3 a 5 & 25 & 45,5 & 79,8 & $( \pm 21,8)$ & 84,1 & $( \pm 18,4)$ & 75,4 & $( \pm 25,3)$ & 79,5 & $( \pm 20,1)$ \\
\hline 6 a 9 & 6 & 10,9 & 85,8 & $( \pm 21,5)$ & 87,6 & $( \pm 21,1)$ & 82,5 & $( \pm 18,2)$ & 86,8 & $( \pm 16,4)$ \\
\hline \multicolumn{11}{|l|}{ Estado civil } \\
\hline Solteira & 22 & 40,0 & 88,9 & $( \pm 16,8)$ & 81,0 & $( \pm 20,3)$ & 78,8 & $( \pm 22,4)$ & 82,2 & $( \pm 18,9)$ \\
\hline Amasiada/Casada & 29 & 52,7 & 79,7 & $( \pm 22,1)$ & 87,1 & $( \pm 14,6)$ & 73,4 & $( \pm 23,3)$ & 79,8 & $( \pm 17,5)$ \\
\hline Divorciada & 4 & 7,3 & 70,0 & $( \pm 19,6)$ & 75,7 & $( \pm 21,9)$ & 76,9 & $( \pm 31,7)$ & 75,0 & $( \pm 23,1)$ \\
\hline \multicolumn{11}{|l|}{ Tem companheiro } \\
\hline Não & 22 & 40,0 & 84,8 & $( \pm 19,4)$ & 79,9 & $( \pm 21,3)$ & 79,7 & $( \pm 23,9)$ & 80,8 & $( \pm 20,1)$ \\
\hline Sim & 33 & 60,0 & 81,2 & $( \pm 21,2)$ & 86,5 & $( \pm 14,5)$ & 73,3 & $( \pm 22,7)$ & 80,1 & $( \pm 17,1)$ \\
\hline \multicolumn{11}{|c|}{ Reside com companheiro } \\
\hline Não & 26 & 47,3 & 85,6 & $( \pm 18,1)$ & 80,4 & $( \pm 20,4)$ & 78,4 & $( \pm 23,2)$ & 81,0 & $( \pm 19,2)$ \\
\hline Sim & 29 & 52,7 & 80,0 & $( \pm 22,2)$ & 86,9 & $( \pm 14,5)$ & 73,5 & $( \pm 23,4)$ & 79,8 & $( \pm 17,6)$ \\
\hline \multicolumn{11}{|l|}{ Tempo relacionamento } \\
\hline Não se aplica & 23 & 41,8 & 85,0 & $( \pm 19,0)$ & 79,5 & $( \pm 20,9)$ & 78,2 & $( \pm 24,4)$ & 80,1 & $( \pm 20,0)$ \\
\hline Até 2 anos & 3 & 5,5 & 88,3 & $( \pm 7,6)$ & 89,5 & $( \pm 13,5)$ & 71,7 & $( \pm 22,7)$ & 81,8 & $( \pm 12,3)$ \\
\hline $2 \mathrm{a} 1 \mathrm{~m}$ a 5 anos & 12 & 21,8 & 90,8 & $( \pm 13,3)$ & 91,2 & $( \pm 12,1)$ & 81,5 & $( \pm 21,4)$ & 87,9 & $( \pm 14,0)$ \\
\hline $5 \mathrm{a} 1 \mathrm{~m}$ a 10 anos & 11 & 20,0 & 68,2 & $( \pm 29,2)$ & 82,3 & $( \pm 16,1)$ & 64,8 & $( \pm 25,9)$ & 72,0 & $( \pm 20,6)$ \\
\hline$>10$ anos & 6 & 10,9 & 80,8 & $( \pm 10,7)$ & 85,7 & $( \pm 16,9)$ & 77,9 & $( \pm 16,0)$ & 81,4 & $( \pm 13,7)$ \\
\hline \multicolumn{11}{|l|}{ № filhos } \\
\hline Nenhum & 22 & 40,0 & 91,4 & $( \pm 12,2)$ & 85,5 & $( \pm 17,6)$ & 80,3 & $( \pm 20,5)$ & 85,0 & $( \pm 14,9)$ \\
\hline 1 a 2 & 26 & 47,3 & 76,7 & $( \pm 24,0)$ & 84,7 & $( \pm 16,7)$ & 73,9 & $( \pm 24,7)$ & 78,5 & $( \pm 20,1)$ \\
\hline 3 a 5 & 7 & 12,7 & 77,1 & $( \pm 19,1)$ & 75,5 & $( \pm 21,8)$ & 68,6 & $( \pm 26,1)$ & 72,9 & $( \pm 19,0)$ \\
\hline Total & 55 & 100,0 & - & & - & & - & & - & \\
\hline
\end{tabular}

A/ISP: Afetivo/Interação Social Positiva; DP: Desvio-padrão; E/l: Emocional/Informação; G: Global; M: Média; Mt: Material.

A Tabela 4 apresenta a percepção de apoio social em função das características clínicas das participantes, sendo importante destacar que não houve diferença significativa entre elas $(p>0,05)$.

\section{Discussão}

O objetivo do presente estudo foi analisar a percepção de apoio social de mulheres no contexto da atenção primária, verificando possível associação com sintomas depressivos e características sociodemográficas, clínicas e de configuração familiar. 
Tabela 4. A percepção de apoio social segundo as características clínicas.

\begin{tabular}{|c|c|c|c|c|c|c|c|c|c|c|}
\hline \multirow{2}{*}{ Variáveis Clínicas } & \multirow{2}{*}{$\mathbf{n}$} & \multirow{2}{*}{$\%$} & \multicolumn{2}{|c|}{ Mt } & \multicolumn{2}{|c|}{ A/ISP } & \multicolumn{2}{|c|}{ E/I } & \multicolumn{2}{|c|}{ G } \\
\hline & & & M & (DP) & M & (DP) & $\mathbf{M}$ & (DP) & M & (DP) \\
\hline \multicolumn{11}{|l|}{ Álcool } \\
\hline Nunca & 33 & 60,0 & 83,9 & $( \pm 17,1)$ & 85,1 & $( \pm 16,5)$ & 78,6 & $( \pm 20,2)$ & 82,1 & $( \pm 15,6)$ \\
\hline 1 vez ou menos/mês & 8 & 14,5 & 68,8 & $( \pm 27,4)$ & 78,6 & $( \pm 20,4)$ & 67,2 & $( \pm 29,4)$ & 71,7 & $( \pm 23,5)$ \\
\hline 2 a 4 vezes/mês & 10 & 18,2 & 87,5 & $( \pm 24,1)$ & 81,4 & $( \pm 19,7)$ & 71,0 & $( \pm 29,2)$ & 78,3 & $( \pm 22,3)$ \\
\hline 2 a 3 vezes/semana & 4 & 7,3 & 87,5 & $( \pm 15,0)$ & 90,0 & $( \pm 20,0)$ & 81,9 & $( \pm 18,4)$ & 88,6 & $( \pm 15,9)$ \\
\hline 4 a 7 vezes/semana & 0 & 0,0 & - & - & - & - & - & - & - & - \\
\hline \multicolumn{11}{|l|}{ Tabaco } \\
\hline Nunca & 79 & 89,1 & 83,3 & $( \pm 20,6)$ & 83,7 & $( \pm 17,8)$ & 76,7 & $( \pm 22,6)$ & 80,9 & $( \pm 17,9)$ \\
\hline 1 a 3 unidades/dia & 1 & 1,8 & 90,0 & - & 71,4 & - & 45,0 & - & 64,2 & - \\
\hline 4 a 10 unidades/dia & 3 & 5,5 & 61,7 & $( \pm 14,4)$ & 80,0 & $( \pm 21,6)$ & 60,0 & $( \pm 32,8)$ & 67,7 & $( \pm 24,2)$ \\
\hline 11 a 20 unidades/dia & 2 & 3,6 & 95,0 & $( \pm 7,1)$ & 100,0 & - & 92,5 & $( \pm 10,6)$ & 95,8 & $( \pm 6,0)$ \\
\hline $21 \mathrm{ou}+$ unidades/dia & 0 & 0,0 & - & - & - & - & - & - & - & - \\
\hline \multicolumn{11}{|l|}{ Exercício físico } \\
\hline 0 a 1 vez/semana & 32 & 58,2 & 83,0 & $( \pm 21,5)$ & 82,1 & $( \pm 19,4)$ & 72,8 & $( \pm 25,4)$ & 78,7 & $( \pm 20,1)$ \\
\hline 2 a 3 vezes/semana & 14 & 25,5 & 78,2 & $( \pm 20,8)$ & 89,2 & $( \pm 16,1)$ & 82,9 & $( \pm 19,7)$ & 84,2 & $( \pm 15,7)$ \\
\hline 4 a 7 vezes/semana & 9 & 16,4 & 88,3 & $( \pm 15,2)$ & 81,9 & $( \pm 12,8)$ & 75,6 & $( \pm 19,8)$ & 80,6 & $( \pm 15,1)$ \\
\hline \multicolumn{11}{|l|}{ Grupos USF } \\
\hline Não & 54 & 98,2 & 82,3 & $( \pm 20,4)$ & 83,8 & $( \pm 17,8)$ & 75,7 & $( \pm 23,4)$ & 80,3 & $( \pm 18,4)$ \\
\hline Sim & 1 & 1,8 & 100,0 & - & 85,7 & - & 82,5 & - & 87,4 & - \\
\hline \multicolumn{11}{|l|}{ Agravos prévios } \\
\hline Não & 34 & 31,8 & 83,1 & $( \pm 19,8)$ & 86,4 & $( \pm 16,1)$ & 78,6 & $( \pm 21,8)$ & 82,4 & $( \pm 16,9)$ \\
\hline Sim & 21 & 38,2 & 81,9 & $( \pm 21,7)$ & 79,7 & $( \pm 19,6)$ & 71,3 & $( \pm 25,2)$ & 77,1 & $( \pm 20,1)$ \\
\hline \multicolumn{11}{|l|}{ Tipos de agravos } \\
\hline Nenhum & 34 & 61,8 & 83,1 & $( \pm 19,8)$ & 86,4 & $( \pm 16,1)$ & 78,6 & $( \pm 21,8)$ & 82,4 & $( \pm 16,9)$ \\
\hline Saúde Mental & 8 & 14,5 & 78,1 & $( \pm 24,2)$ & 83,2 & $( \pm 18,5)$ & 73,8 & $( \pm 26,6)$ & 78,2 & $( \pm 20,3)$ \\
\hline Saúde Mental + Outros & 7 & 12,7 & 77,9 & $( \pm 23,2)$ & 70,2 & $( \pm 21,8)$ & 65,7 & $( \pm 27,5)$ & 69,9 & $( \pm 21,0)$ \\
\hline Outros & 6 & 10,9 & 91,7 & $( \pm 16,0)$ & 86,0 & $( \pm 17,2)$ & 74,6 & $( \pm 23,9)$ & 84,1 & $( \pm 19,4)$ \\
\hline Total & 55 & 100,0 & - & & - & & - & & - & \\
\hline
\end{tabular}

A/ISP: Afetivo/Interação Social Positiva; DP: Desvio-padrão; E/l: Emocional/Informação; G: Global; M: Média; Mt: Material.

Os resultados relativos aos escores médios encontrados nas três dimensões de apoio social se assemelham a outro estudo nacional ${ }^{6}$ e, embora o instrumento utilizado em sua avaliação não apresente categorias quanto ao nível de percepção de apoio social (por exemplo em alta, moderada e baixa), podemos considerá-los relativamente elevados. No que se refere à ocorrência de sintomas depressivos, apesar da diversidade metodológica e amostral dos estudos, resultados aqui encontrados corroboram os achados nacionais ${ }^{15-19}$ e internacionais. ${ }^{20-25}$

A associação negativa entre apoio social e sintomas depressivos evidenciada neste estudo encontra uma base sólida na literatura nacional e internacional, principalmente em estudos com gestantes e puérperas, as quais se assemelham em termos da faixa-etária da amostra estudada. ${ }^{26,27}$ Uma pesquisa norte-americana sobre depressão pós-parto demonstrou que mulheres que relataram possuir dois ou mais amigos/parentes com quem pudessem contar apresentaram menor escore de depressão em relação àquelas que possuíam apenas uma ou nenhuma pessoa próxima. ${ }^{28}$ No Brasil, um estudo observou que 
quanto maior a pontuação na escala de percepção de suporte social, menor a pontuação na escala de depressão pós-parto de Edinburgh. ${ }^{29}$

Também foram encontrados resultados semelhantes em estudos com mulheres afro-americanas residentes em Detroit, ${ }^{30}$ adolescentes norte-americanas, ${ }^{31}$ idosos brasileiros de ambos os sexos, ${ }^{32}$ noruegueses de ambos os sexos entre 20 e 89 anos $^{33}$ e holandeses de ambos os sexos entre 13 e 25 anos. ${ }^{34}$ Estas evidências devem servir de alerta para os profissionais da atenção primária ao conduzirem suas avaliações de rotina, de modo a não negligenciar a influência da percepção de apoio social sobre a depressão e vice-versa.

Com relação aos achados entre as dimensões de apoio social e a variável renda, resultados semelhantes sugerem que indivíduos com poder aquisitivo maior tendem a ser mais independentes e autossuficientes em sua vida social, por isso, em situações de real necessidade percebem-se mais apoiados. ${ }^{6}$ Os resultados encontrados entre o estado civil e a percepção de apoio social Material, por sua vez, contrariam os achados da literatura nacional, ${ }^{6}$ podendo tal divergência estar relacionada com o expressivo crescimento da participação feminina no sustento do lar, de 22,2\% em 2000 para 37,3\% em 2010, colocando-as em uma posição de oferecer o suporte financeiro/material ao invés de recebê-lo. ${ }^{35}$

Considerando o número de filhos e a percepção de apoio social, os resultados corroboram os achados de um estudo brasileiro no qual quanto maior o número de filhos relatado pelas participantes com idade entre 20 e 43 anos residentes no interior oeste do Estado de Santa Catarina, menor a percepção de apoio social nas dimensões Material $(r=0,28 ; p<0,05)$, Afetivo/Interação Social Positivo $(r=0,38 ; p<0,01)$ e Emocional/Informação $(r=0,32 ; p<0,05)$. Os autores apontam que o maior número de filhos pode favorecer um aumento na busca por apoio, o que pode gerar ou não a resposta dos parentes próximos e amigos a esse pedido. $\mathrm{E}$ ainda que haja uma resposta positiva, esse retorno pode não ser considerado satisfatório devido ao número de tarefas exigidas pela maternidade. ${ }^{36}$

A ausência de associações entre a percepção de apoio social e as características clínicas diverge de outro estudo nacional, o qual observou maior percepção de apoio social em participantes que praticavam atividades físicas/artísticas ou trabalho voluntário. ${ }^{6} \mathrm{~A}$ distribuição heterogênea das participantes entre as categorias de cada variável pode estar associada a este resultado inesperado.

Apesar do esforço deste estudo em enriquecer as discussões sobre o tema, cabe ressaltar que o tamanho reduzido e conveniente da amostra foram suas principais limitações, inviabilizando a generalização dos resultados para grupos de mulheres com características diferentes das apresentadas pela amostra. Além disso, a utilização de um instrumento de rastreamento permite afirmar apenas a presença de sintomas depressivos, sendo sugerido para futuras pesquisas o uso de avaliações diagnósticas que confirmem ou refutem a hipótese de transtornos do humor.

\section{Conclusão}

O ser humano se desenvolve em um contexto social mediado por diferentes relações ao longo da vida que podem ou não ser fontes de apoio e cuidado. Em vista disto, a associação entre vulnerabilidade no apoio social e problemas de saúde mental em mulheres sinaliza o quanto as equipes de saúde da atenção primária precisam estar atentas também à faixa etária adulto-jovem por se tratar de um período em que as exigências sociais se acumulam frente às atividades ocupacionais, acadêmicas e familiares. 
Analisar a percepção do apoio social e a sintomatologia depressiva em mulheres jovens, conforme a proposta deste estudo, mostra-se relevante ao demonstrar que, independentemente vulnerabilidade específica (exemplo: gestantes e puérperas com bebês internados), estas mulheres requerem cuidado e acompanhamento mais próximo, pois podem estar em sofrimento velado. Essas informações podem ampliar o olhar e favorecer o desenvolvimento de ações de promoção à saúde no sentido de fortalecer as relações na comunidade, sobretudo o papel das unidades de saúde da família como uma fonte de apoio/suporte social.

\section{Referências}

1. Andrade CR. Associação entre apoio social e frequência relatada de auto-exame das mamas no Estudo Pró-Saúde [Dissertação de mestrado]. Rio de Janeiro: Escola Nacional de Saúde Pública Sérgio Arouca, Fundação Oswaldo Cruz; 2004. 67 p.

2. Bowling A. Measuring social networks and social support. In: Bowling A, ed. Measuring health: a review of quality of life measurements scales. 2nd ed. Buckingham: Open University Press; 1997. p. 91-109.

3. Sherbourne CD, Stewart AL. The MOS social support survey. Soc Sci Med. 1991;32(6):705-14. DOI: http://dx.doi. org/10.1016/0277-9536(91)90150-B

4. Liu DG, Wang SS, Peng RJ, Qin T, Shi YX, Teng XY, at al. Interaction of social support and psychological stress on anxiety and depressive symptoms in breast cancer patients. Asian Pac J Cancer Prev. 2011;12(10):2523-9.

5. Due P, Holstein B, Lund R, Modvig J, Avlund K. Social relations: network, support and relational strain. Soc Sci Med. 1999;48(5):661-73. DOI: http://dx.doi.org/10.1016/S0277-9536(98)00381-5

6. Griep RH, Chor D, Faerstein E, Werneck GL, Lopes CS. Validade de constructo de escala de apoio social do Medical Outcomes Study adaptada para o português no Estudo Pró-Saúde. Cad Saúde Pública. 2005;21(3):703-14. DOI: http:// dx.doi.org/10.1590/S0102-311X2005000300004

7. Thiengo DL, Santos JFC, Mason VC, Abelha L, Lovisi GM. Associação entre apoio social e depressão durante a gestação: uma revisão sistemática. Cad Saúde Colet. 2011;19(2):129-38.

8. American Psychiatric Association (APA). Manual Diagnóstico e Estatístico de Transtornos Mentais. 5a ed. Porto Alegre: Artmed; 2014. $992 \mathrm{p}$.

9. Villano LAB, Nanhay ALG. Depressão: epidemiologia e abordagem em cuidados primários da saúde. Rev Hosp Univ Pedro Ernesto. 2011;10(2):10-20.

10. Kaplan HI, Sadock BJ, Greb JA. Compêndio de psiquiatria: ciências do comportamento e psiquiatria clínica. 7a ed. Porto Alegre: Artmed; 1997.

11. Linde K, Willich SN. How objective are systematic reviews? Differences between reviews on complementary medicine. J R Soc Med. 2003;96(1):17-22. PMID: 12519797 DOI: http://dx.doi.org/10.1258/jrsm.96.1.17

12. Beck AT, Ward $\mathrm{CH}$, Mendelson M, Mock J, Erbaugh J. An inventory for measuring depression. Arch Gen Psychiat. 1961;4:561-71. DOI: http://dx.doi.org/10.1001/archpsyc.1961.01710120031004

13. Cunha JA. Manual da versão em português das Escalas Beck. São Paulo: Casa do Psicólogo; 2001.

14. Cohen J. Statistical power analysis for the behavioral sciences. 2nd ed. New York: Academic Press; 1988.

15. Bromberger JT. A psychosocial understanding of depression in women: for the primary care physician. J Am Med Womens Assoc. 2004;59(3):198-206.

16. Ribeiro Dos Santos E, Huang H, Menezes PR, Scazufca M. Prevalence of Depression and Depression Care for Populations Registered in Primary Care in Two Remote Cities in the Brazilian Amazon. PLoS One. 2016;11(3):e0150046. DOI: http:// dx.doi.org/10.1371/journal.pone.0150046

17. Gonçalves DA, Mari Jde J, Bower P, Gask L, Dowrick C, Tófoli LF, et al. Brazilian multicentre study of common mental disorders in primary care: rates and related social and demographic factors. Cad Saúde Pública. 2014;30(3):623-32. DOI: http://dx.doi.org/10.1590/0102-311X00158412 
18. Gonçalves DM, Kapczinski F. Prevalência de transtornos mentais em indivíduos de uma unidade de referência para Programa Saúde da Família em Santa Cruz do Sul, Rio Grande do Sul, Brasil. Cad Saúde Pública. 2008;24(9):2043-53. DOI: http://dx.doi.org/10.1590/S0102-311X2008000900010

19. Molina MRAL, Wiener CD, Branco JC, Jansen K, Souza LDM, Tomasi E, et al. Prevalência de depressão em usuários de unidades de atenção primária. Rev Psiquiatr Clín. 2012;39(6):194-7. DOI: http://dx.doi.org/10.1590/S010160832012000600003

20. Coyne JC, Fechner-Bates S, Schwenk TL. Prevalence, nature, and comorbidity of depressive disorders in primary care. Gen Hosp Psychiatry. 1994;16(4):267-76. PMID: 7926703 DOI: http://dx.doi.org/10.1016/0163-8343(94)90006-X

21. Mergl R, Seidscheck I, Allgaier AK, Möller HJ, Hegerl U, Henkel V. Depressive, anxiety, and somatoform disorders in primary care: prevalence and recognition. Depress Anxiety. 2007;24(3):185-95. DOI: http://dx.doi.org/10.1002/da.20192

22. Janosky JE, South-Paul JE, Lin CJ. Pain and depression in a cohort of underserved, community-dwelling primary care patients. J Am Board Fam Med. 2012;25(3):300-7. DOI: http://dx.doi.org/10.3122/jabfm.2012.03.080138

23. Milanović SM, Erjavec K, Poljičanin T, Vrabec B, Brečić P. Prevalence of depression symptoms and associated sociodemographic factors in primary health care patients. Psychiatr Danub. 2015;27(1):31-7.

24. Holden KB, Bradford LD, Hall SP, Belton AS. Prevalence and correlates of depressive symptoms and resiliency among African American women in a community-based primary health care center. J Health Care Poor Underserved. 2013;24(4 Suppl):79-93.

25. Wong ST, Manca D, Barber D, Morkem R, Khan S, Kotecha J, et al. The diagnosis of depression and its treatment in Canadian primary care practices: an epidemiological study. CMAJ Open. 2014;2(4):E337-42. DOI: http://dx.doi.org/10.9778/ cmajo.20140052

26. Cankorur VS, Abas M, Berksun O, Stewart R. Social support and the incidence and persistence of depression between antenatal and postnatal examinations in Turkey: a cohort study. BMJ Open. 2015;5(4):e006456. DOI: http://dx.doi. org/10.1136/bmjopen-2014-006456

27. Ren J, Jiang X, Yao J, Li X, Liu X, Pang M, et al. Depression, Social Support, and Coping Styles among Pregnant Women after the Lushan Earthquake in Ya'an, China. PLoS One. 2015;10(8):e0135809. DOI: http://dx.doi.org/10.1371/journal. pone.0135809

28. Surkan PJ, Peterson KE, Hughes MD, Gottlieb BR. The role of social networks and support in postpartum women's depression: a multiethnic urban sample. Matern Child Health J.2006;10(4):375-83. DOI: http://dx.doi.org/10.1007/s10995005-0056-9

29. Baptista MN, Baptista ASD, Torres ECR. Associação entre suporte social, depressão e ansiedade em gestantes. Psic. 2006;7(1):39-48.

30. Israel BA, Farquhar SA, Schulz AJ, James SA, Parker EA. The relationship between social support, stress, and health among women on Detroit's East Side. Health Educ Behav. 2002;29(3):342-60.

31. Stice E, Ragan J, Randall P. Prospective relations between social support and depression: differential direction of effects for parent and peer support? J Abnorm Psychol. 2004;113(1):155-9. PMID: 14992668

32. Carneiro RS, Falcone E, Clark C, Del Prette Z, Del Prette A. Qualidade de vida, apoio social e depressão em idosos: relação com habilidades sociais. Psicol Reflex Crít. 2007;20(2):229-37.

33. Grav S, Hellzèn O, Romild U, Stordal E. Association between social support and depression in the general population: the HUNT study, a cross-sectional survey. J Clin Nurs. 2012;21(1-2):111-20. DOI: http://dx.doi.org/10.1111/j.13652702.2011.03868.x

34. Landman-Peeters KM, Hartman CA, van der Pompe G, den Boer JA, Minderaa RB, Ormel J. Gender differences in the relation between social support, problems in parent-offspring communication, and depression and anxiety. Soc Sci Med. 2005;60(11):2549-59. DOI: http://dx.doi.org/10.1016/j.socscimed.2004.10.024

35. Brasil. Instituto Brasileiro de Geografia e Estatística - IBGE. Censo demográfico 2010. Famílias e Domicílios. Resultados da amostra. [Acesso 24 Jan 2016]. Disponível em: http://www.censo2010.ibge.gov.br/

36. Vieira V, Vieira ML, Prado AB. Apoio social: percepção materna em contextos com diferentes graus de urbanização. Estud Psicol (Natal). 2011;16(3):209-17. 